\title{
Controlled gentamicin release from multi-layered electrospun nanofibrous structures of various thicknesses
}

This article was published in the following Dove Press journal:

International Journal of Nanomedicine

5 October 2012

Number of times this article has been viewed

\author{
Jakub Sirc' \\ Sarka Kubinova ${ }^{2}$ \\ Radka Hobzova' \\ Denisa Stranska ${ }^{3}$ \\ Petr Kozlik ${ }^{4}$ \\ Zuzana Bosakova ${ }^{4}$ \\ Dana Marekova ${ }^{2}$ \\ Vladimir Holan ${ }^{5}$ \\ Eva Sykova ${ }^{2}$ \\ Jiri Michalek' \\ 'Department of Polymer Gels, \\ Institute of Macromolecular \\ Chemistry, Academy of Science \\ of the Czech Republic, Prague, \\ Czech Republic; ${ }^{2}$ Department \\ of Neuroscience, Institute of \\ Experimental Medicine, Academy \\ of Science of the Czech Republic, \\ Prague, Czech Republic; ${ }^{3}$ Elmarco Ltd, \\ Liberec, Czech Republic; ${ }^{4}$ Department \\ of Analytical Chemistry, Faculty \\ of Science, Charles University in \\ Prague, Prague, Czech Republic; \\ ${ }^{5}$ Department of Transplant \\ Immunology, Institute of Molecular \\ Genetics, Academy of Science \\ of the Czech Republic, Prague, \\ Czech Republic
}

Correspondence: Jakub Sirc

Institute of Macromolecular Chemistry,

Academy of Science of the Czech

Republic, Heyrovsky Sq 2,

16206 Prague 6, Czech Republic

$\mathrm{Tel}+420296809270$

Fax +420296809 410

Email sirc@imc.cas.cz

\begin{abstract}
Polyvinyl alcohol nanofibers incorporating the wide spectrum antibiotic gentamicin were prepared by Nanospider ${ }^{\mathrm{TM}}$ needleless technology. A polyvinyl alcohol layer, serving as a drug reservoir, was covered from both sides by polyurethane layers of various thicknesses. The multilayered structure of the nanofibers was observed using scanning electron microscopy, the porosity was characterized by mercury porosimetry, and nitrogen adsorption/desorption measurements were used to determine specific surface areas. The stability of the gentamicin released from the electrospun layers was proved by high-performance liquid chromatography (HPLC) and inhibition of bacterial growth. Drug release was investigated using in vitro experiments with HPLC/MS quantification, while the antimicrobial efficacy was evaluated on Gram-positive Staphylococcus aureus and Gram-negative Pseudomonas aeruginosa. Both experiments proved that the released gentamicin retained its activity and showed that the retention of the drug in the nanofibers was prolonged with the increasing thickness of the covering layers.
\end{abstract}

Keywords: nanofibers, electrospinning, multilayered structure, morphology, gentamicin, drug release

\section{Introduction}

The highly porous structure of nanofibers makes them attractive materials for various biomedical applications. Numerous reports have described the fabrication of electrospun polymer nanofibers applicable in tissue engineering for the regeneration of skin, bone, heart, cornea, the nervous system, and other tissues. ${ }^{1-3}$ The specific morphology of nanofibrous nonwoven materials makes them suitable for wound dressings. ${ }^{3,4}$ Their high porosity, consisting of relatively small pores, enables good permeability for oxygen and water but protects wounds from dehydration and bacterial penetration. ${ }^{3,5}$ Moreover, the large surface area and high pore volume of nanofibers promote the migration of keratinocytes on the wound surface and may play a role in accelerating the healing process. Nanofibers composed of natural polymers, such as chitin, ${ }^{6}$ chitosan $^{7}$ or collagen, ${ }^{8}$ or synthetic polymers or their blends, such as polyurethane (PUR), ${ }^{9,10}$ polyvinyl alcohol (PVA),${ }^{10}$ polyacrylonitrile, ${ }^{10}$ or polyethersulfone,${ }^{11}$ have been specifically engineered as dressings for wound healing or as tissue engineered scaffolds for skin substitutes. In addition to physical protection of the wound site, nanofibrous materials have also been developed for the local delivery of therapeutic agents, such as antibiotics, ${ }^{12}$ anesthetics, ${ }^{13,14}$ or growth factors. ${ }^{7,15}$

Various processing techniques have been used to produce nanofibers. ${ }^{1,16-18}$ However, the electrospinning technique allows the production of continuous polymeric nanofibers and provides numerous opportunities to modify and control their 
morphological parameters, such as surface area, fiber diameter, the porosity of the nanofibrous layer (fiber density), basis weight, ${ }^{19}$ or internal fiber structure. ${ }^{18,20,21}$ Nanospider ${ }^{\mathrm{TM}}$ electrospinning technology is based on a needleless method in which polymeric jets are spontaneously formed from liquid surfaces $^{22,23}$ and allows the production of fibers with diameters ranging from tens of nanometers to tens of micrometers. Nanospider ${ }^{\mathrm{TM}}$ (Elmarco, Liberec, Czech Republic) technology is very flexible, enabling the formation of nanofibrous materials from various polymers. ${ }^{24}$ The process provides high production capacity, stability, and easy maintenance compared to other known industrial-scale technologies based on needles or capillary spinners. ${ }^{25,26}$ The incorporation of various additional substances, such as pharmacologically active compounds, in the nanofibers can be accomplished by simply adding the substances to the polymer solution. In our previous work ${ }^{27}$ using Nanospider ${ }^{\mathrm{TM}}$ technology, we demonstrated that the immunosuppressive drug cyclosporine A can be incorporated directly into electrospun nanofibers without any loss of its pharmacological activity.

To achieve drug release from nanofibers, two basic delivery designs are known: matrices and reservoirs. In the matrix-type of carriers, the drug is homogeneously dispersed in the material of the nanofiber, and its release is based on solid-state diffusion or a desorption mechanism; ${ }^{13,28,29}$ however, such drug-loaded systems tends to have a strong burst release within the first hours. In the second design, reservoir-type structures are formed by a drug-loaded core and a covering polymer shell. ${ }^{21,28,30}$ The core-shell structure enables better control of the drug release profile by adjusting the shell's properties, such as its microstructure, thickness, and degradability. On the other hand, some of the complications that persist during nanofiber preparation do not allow for easy large-scale production.

Several papers have described the preparation and testing of multi-layer polymer nanofibrous structures containing a middle layer that incorporates a pharmacologically active compound, covered by other nanofibrous layers of a different polymer. ${ }^{31-33}$ Chen et al $^{31}$ prepared biodegradable sandwichstructured nanofibers from poly(D,L)-lactide-co-glycolide and collagen with incorporated vancomycin, gentamicin, and lidocaine. They described the conditions of nanofibrous mat preparation by classical jet electrospinning and the in vitro release of the drugs, as well as their effect on the in vivo healing process in rat wounds. Kim et $\mathrm{al}^{32}$ observed rhodamine and peptide release from multi-layered PCL/polyethylene oxide/PCL nanofibers. They found a decrease of the initial burst release with increasing thickness of the covering layer and a slower release of compounds from sandwiches with thicker covering layers. Nevertheless, all of these authors prepared nanofibers by classical electrospinning based on needle or capillary spinners.

In contrast, needleless electrospinning allows the sequential deposition of nanofibrous layers, and therefore, the preparation of compact sandwich structures in which the middle drug-loaded layer is covered by layers of a different polymer. These layers, with a large surface area and a microporous structure, improve the mechanical properties, promote wound healing, and also contribute to controlled drug release to the surrounding tissue. Combining more layers of the required composition, porosity, thickness, fiber diameter, and/or drug concentration, it is possible to obtain a nanofibrous material with properties suitable for specific applications.

The aim of this study was to employ needleless electrospinning for the preparation of multi-layer nanofibrous structures consisting of a drug-loaded middle layer and covering layers of various thicknesses, enabling the controlled release of the drug. The middle layer was made from crosslinked or noncrosslinked PVA nanofibers containing the wide spectrum antibiotic gentamicin, while the covering layers were made from PUR. The morphology of the prepared nanofibers was observed using scanning electron microscopy (SEM) and characterized by Brunauer, Emmet, and Teller (BET) nitrogen adsorption/desorption measurements and mercury porosimetry. Gentamicin release was quantified in vitro using high-performance liquid chromatography/mass spectrometry (HPLC/MS), and its bactericidal effect was confirmed on Staphylococcus aureus and Pseudomonas aeruginosa cultures. The effect of the preparation conditions on the morphology of the nanofibers and subsequent gentamicin release was evaluated.

\section{Materials and methods}

Polyvinyl alcohol (PVA) (type Z 220, viscosity of $4 \mathrm{wt} \%$ water solution at $20^{\circ} \mathrm{C} 11.5-15 \mathrm{mPa}$.s, saponification degree 90.5-92.5 mol\%) was supplied by Nippon Gohsei (Osaka, Japan). PUR (Estane 5714 F1, viscosity of $15 \mathrm{wt} \%$ solution in THF 600-900 mPa.s) was obtained from Lubrizol Corp (Wickliffe, OH). Gentamicin was purchased from AppliChem GmbH (Darmstadt, Germany). N,N-Dimethylacetamide (Sigma-Aldrich, St Louis, MO) and toluene and phosphoric acid (Penta a.s., Prague, Czech Republic) were obtained in analytical reagent grade and used as received.

PVA and PUR nanofibers were prepared using Nanospider ${ }^{\mathrm{TM}}$ technology. ${ }^{22}$ The process parameters were individually optimized for each polymer. PVA nanofibrous 
layers were made at a basis weight of $0.8 \mathrm{~g} / \mathrm{m}^{2}$, while the covering PUR layers had basis weights of $1.5,4.4$, and $14.7 \mathrm{~g} / \mathrm{m}^{2}$. Layers of each polymer intended for mercury porosimetry and nitrogen adsorption/desorption measurements were prepared separately in thicknesses (basis weights) similar to the sandwich structures.

PVA was dissolved in a water/phosphoric acid mixture at a polymer concentration of $11 \mathrm{wt} \%$. The distance between the spinning electrode and the collector was $13 \mathrm{~cm}$; the spinning electrode rotated at $2 \mathrm{rpm}$, and the high voltage supply was $45-55 \mathrm{kV} / \mathrm{cm}$. The relative humidity was $25 \%-30 \%$ and the temperature $22^{\circ} \mathrm{C}$. Gentamicin, at a concentration of $10 \mathrm{wt} \%$, was added directly to the PVA electrospun mixture. The PVA layers were crosslinked thermally in a drying oven at $145^{\circ} \mathrm{C}$ for 15 minutes.

PUR was dissolved in an $N, N$-dimethylacetamide/toluene mixture at a ratio of $2: 1(\mathrm{w} / \mathrm{w})$ and a polymer concentration of $10 \mathrm{wt} \%$. The electrospinning conditions were: $4 \mathrm{rpm}$ for the spinning electrode, a $15 \mathrm{~cm}$ distance between the electrodes, and a $5 \mathrm{kV} / \mathrm{cm}$ electric field strength. The relative humidity was kept lower than $30 \%$ due to the risk of solvent explosion.

The PUR/PVA/PUR multi-layered sandwich structures were prepared by multi-step deposition of each layer. All layers were prepared on the same Nanospider ${ }^{\mathrm{TM}}$ machine; only the process parameters were varied according to the polymer type as described above. Nonwoven polypropylene material (ATEX, Milan, Italy) was used as a support for the deposition of the first PUR layer. The different thickness of each layer $\left(\mathrm{g} / \mathrm{m}^{2}\right)$ results from the speed of the rotating collecting electrode. In the case of the PVA layer with a basis weight of $0.8 \mathrm{~g} / \mathrm{m}^{2}$, the speed was $50 \mathrm{~mm} / \mathrm{min}$; for the PUR layers, the speeds were as follows: $1.5 \mathrm{~g} / \mathrm{m}^{2}=110 \mathrm{~mm} / \mathrm{min}$, $4.4 \mathrm{~g} / \mathrm{m}^{2}=30 \mathrm{~mm} / \mathrm{min}$, and $14.7 \mathrm{~g} / \mathrm{m}^{2}=18 \mathrm{~mm} / \mathrm{min}$ with double electrospinning.

The structure of the prepared nanofibers was observed using a TS 5130 VEGA scanning electron microscope, TESCAN (Brno, Czech Republic). Multilayer samples were fractured in liquid nitrogen and sputtered with a gold layer. The fiber diameters and the thicknesses of the nanofibrous layers were determined as mean values of 30 measurements in various locations. Single layers of PVA and PUR nanofibers were prepared to determine porosity and surface area. Mercury porosimetry measurements were made using an Autopore IV 9500 porosimeter (Micromeritics, Norcross, GA), and specific surface areas were calculated from nitrogen absorption/desorption isotherms recorded on an ASAP 2020 apparatus (Micromeritics).
Gentamicin release from the nanofibrous mats was investigated under laboratory conditions and by in vitro bacteriological assay. Laboratory experiments were performed on agar dishes (Caso-Agar, Mercoplate ${ }^{\circledR}$; Merck and Co, Whitehouse Station, NJ) in order to evaluate the time course of the drug release from the nanofibrous mats. Discs of $8 \mathrm{~mm}$ in diameter were cut from the nanofibrous mats and placed on agar previously wetted with $0.5 \mathrm{~mL}$ of distilled water, 1 day before the release experiment. After various time periods, the discs were removed, immersed into distilled water ( $1 \mathrm{~mL}$ each) and shaken for 2 days to rinse out the remaining gentamicin. The concentration of gentamicin in solution was quantified by an HPLC-MS Liquid Chromatograph Series 1200 with a Triple Quad LC/ MS 6460 tandem mass spectrometer (Agilent Technologies, Waldbroon, Germany). The amount of gentamicin released into the agar was calculated as the difference between the initial and residual amounts of gentamicin in the disc. The drug release from each nanofibrous sample was determined 15 times and the average values given. The HPLC-MS apparatus was also used to evaluate the stability of gentamicin during the electrospinning process.

The in vitro antimicrobial properties of electrospun layers containing gentamicin were evaluated from inhibitory zone measurements against the Gram-positive organism Staphylococcus aureus (ATCC 6538) and the Gram-negative organism Pseudomonas aeruginosa (ATCC 27853). The bacterial strains were obtained from the Czech Collection of Microorganisms, Brno, Czech Republic. A gelatin pellet infused with the bacterial strain was incubated in $9 \mathrm{~mL}$ of liquid media (Tryptic Soy Broth, Mecrotube ${ }^{\circledR}$; Merck and Co) at $37^{\circ} \mathrm{C}$ for 24 hours, and then diluted 1000 -fold in a phosphate buffer. An aliquot of the suspension $(0.7 \mathrm{~mL})$ was uniformly spread onto an agar plate (Caso-Agar, Mercoplate ${ }^{\circledR}$; Merck and $\mathrm{Co}$ ) and allowed to dry for several minutes. The nanofibrous mats (6-mm diameter discs) were placed on the agar plates and incubated at $37^{\circ} \mathrm{C}$. The zones of inhibition were measured after 1, 2, and 6 days.

The release profiles of the gentamicin from the nanofibrous layered mats were evaluated using the inhibition of S. aureus after 1, 3, 6, 24, and 48 hours of treatment. Upon removal from the agar plate, the nanofibrous discs were placed on a new agar plate to further evaluate the inhibitory effect from the residual gentamicin in the sample. The plates were incubated overnight at $37^{\circ} \mathrm{C}$, and the zones of inhibition were measured from photomicrographs using ImageJ software. To eliminate potential variability between the samples during the manufacturing process, the area of inhibition 
was calculated as (area of inhibition/area of inhibition after 48 hours $) \times 100$, as the area of inhibition after 48 hours was found to be maximal; also, no residual inhibitory activity was detected in the samples after 48 hours of drug release. Measurements of the inhibitory zones were repeated four times for each nanofiber sample. Antimicrobial susceptibility testing discs (Oxoid, Cambridge, UK) containing 10, 30, or $120 \mu$ of gentamicin were used as standards.

\section{Results and discussion}

Preparation and morphological characterization of nanofibrous mats

All nanofibrous layers were made using Nanospider ${ }^{\mathrm{TM}}$ technology. The multilayered nanofibrous mats were prepared in a multi-step process, changing the process parameters and evaluating the area weight after each step.

The structures of the prepared nanofibrous mats were observed using SEM. The images did not reveal any artifacts or heterogeneities, and uniform nanofibrous structures were found. From the SEM images, the fiber diameters were calculated as the mean value of 30 measurements at various locations. The mean fiber diameter obtained for PVA fibers was $185 \pm 70 \mathrm{~nm}$, while in the case of PUR fibers it was $420 \pm 150 \mathrm{~nm}$. The fiber diameters differed between PVA and PUR nanofibers; however, the diameters were similar for samples of the same polymer, regardless of the layer thickness. This is due to the method of preparation, by which the fiber thickness is particularly influenced by the viscosity of the solution, the strength of the electrostatic field, and the distance between the electrodes; the mat's thickness is driven by the speed of rotation of the collecting electrode and the duration of fiber deposition, while the other parameters remain unchanged.

The structures of the three-layered nanofibrous PUR/ PVA/PUR mats evaluated by SEM are depicted in Figure 1. It can be seen that fracturing the samples in liquid nitrogen successfully maintained the inner sandwich structure and the original thickness of each layer. On the other hand, some secondary changes in the nanofibers, especially local melting of the fibers, can be seen. The mean values of the layer thicknesses calculated from the SEM measurements are given in Table 1 . The values obtained showed a proportional increase similar to the increasing basis weights. It should be mentioned that the actual thickness of the nanofibrous layers in the subsequent drug release experiments, as well as in their potential in vivo application, will certainly differ from the values obtained by SEM in a vacuum/dry state, due to the absorption of water under aqueous conditions.
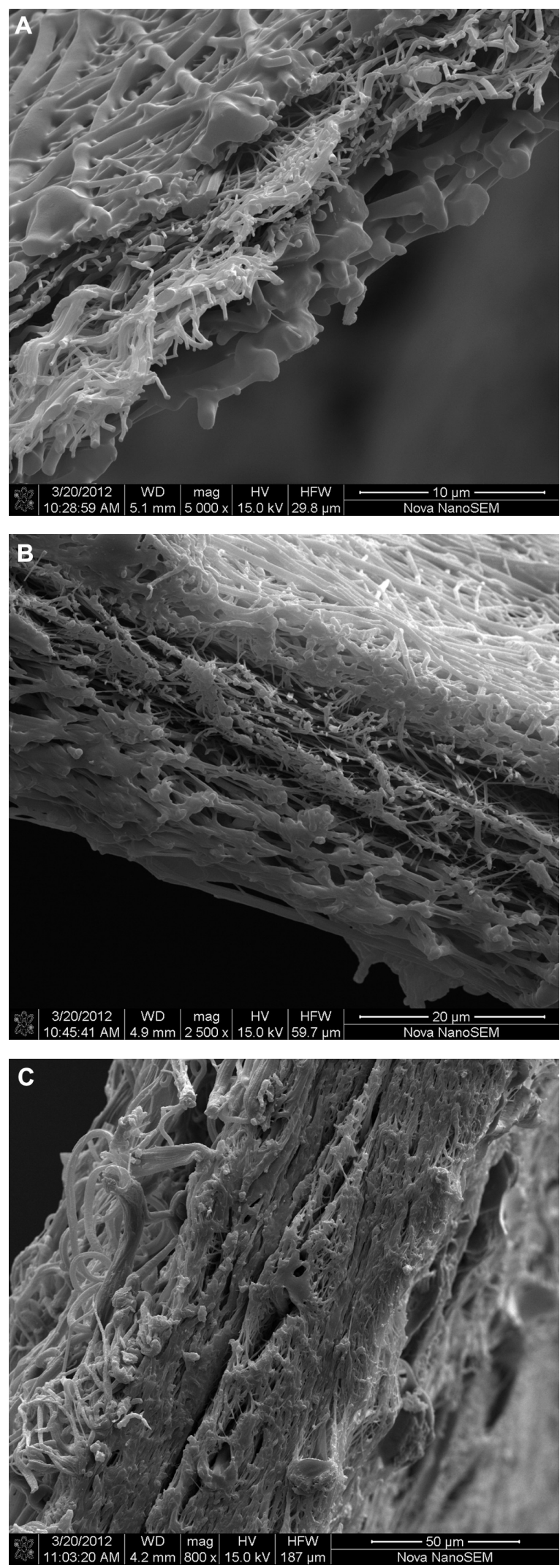

Figure I SEM images of multilayer nanofibrous structures in which the middle crosslinked PVA layer $\left(0.8 \mathrm{~g} / \mathrm{m}^{2}\right)$ containing $10 \mathrm{wt} \%$ gentamicin is covered with PUR layers of 1.5 (A), 4.4 (B), or $14.7 \mathrm{~g} / \mathrm{m}^{2}$ (C) basis weight.

Abbreviations: PUR, polyurethane; PVA, polyvinyl alcohol; SEM, scanning electron microscopy. 
Table I Morphological characteristics of the prepared nanofibers

\begin{tabular}{lllll}
\hline Polymer & $\begin{array}{l}\text { Basis weight } \\
\left(\mathrm{g} / \mathbf{m}^{2}\right)^{\mathbf{a}}\end{array}$ & $\begin{array}{l}\text { Layer thickness } \\
(\mu \mathrm{m})^{\mathbf{a}}\end{array}$ & $\begin{array}{l}\text { Surface area } \\
\left(\mathbf{m}^{2} / \mathbf{g}\right)^{\mathrm{b}}\end{array}$ & $\begin{array}{l}\text { Porosity } \\
(\%)^{\mathrm{b}}\end{array}$ \\
\hline PVA $^{\mathrm{C}}$ & 0.8 & $3.1 \pm 0.6$ & 12.8 & 85.8 \\
PUR & 1.5 & $3.4 \pm 1.0$ & 7.5 & 78.9 \\
PUR & 4.4 & $8.1 \pm 1.1$ & 9.1 & 76.8 \\
PUR & 14.7 & $26.3 \pm 3.7$ & 8.5 & 70.7 \\
\hline
\end{tabular}

Notes: ameasured on multilayered structures of PUR/PVA/PUR mats; ${ }^{b}$ measured on single PVA and PUR layers; 'the PVA layer was thermally crosslinked. Abbreviations: PUR, polyurethane; PVA, polyvinyl alcohol.

Nitrogen adsorption/desorption BET measurements and mercury porosimetry were carried out with single PVA and PUR layers of the same basis weight as in the sandwich structures, and the results obtained are summarized in Table 1. BET measurements of specific surface areas revealed higher values in the case of the PVA fibers $\left(12.8 \mathrm{~m}^{2} / \mathrm{g}\right)$ compared to the PUR ones (between $7.5 \mathrm{~m}^{2} / \mathrm{g}$ and $9.1 \mathrm{~m}^{2} / \mathrm{g}$ ), which corresponds to the smaller diameter of the PVA fibers. Similar to the fiber diameters, comparing PUR layers of various thicknesses revealed that the surface areas were not influenced by the basis weight.

The porosity of the PVA nanofibers $(85.8 \%)$ determined by mercury porosimetry was higher than the porosity of the PUR nanofibrous samples (70.7\%-78.9\%; see Table 1). The porosity of the PUR layers slightly decreased with increasing basis weight, which was caused by the preparation procedure in which the fibers were deposited stepwise for a certain length of time. With the increasing deposition time needed to obtain thicker layers, a certain level of compression of a whole layer can occur, ie, the formed nanofibrous layer is more compact. The total pore volume of such layers consequently becomes lower. Mercury porosimetry, as well as BET measurements, proceeds on single layers, and therefore, the obtained values may be slightly different from those of real sandwich structures. Nevertheless, the measurements of single layers provided us with more detailed information about morphological parameters depending on polymer type or layer thickness. An important finding was that SEM images of the sandwich structures did not show any noticeable difference between the upper and lower PUR nanofibrous layers (see Figure 1), so the stepwise deposition of subsequent layers did not cause any significant compression of the lower layers.

Pore size distribution curves (not depicted) obtained from mercury porosimetry measurements did not show any pores less than tens of nanometers in size, suggesting that there is no porosity inside the PUR and PVA fibers and that the whole pore volume is given by the inter-fiber space. The relatively low values of specific surface areas (see Table 1) obtained by BET measurements also correspond to this finding.

No morphological differences were observed between samples consisting of crosslinked or noncrosslinked PVA layers. It can be noted that the additional crosslinking of the PVA layers had no effect on any structural parameters of the resulting nanofibrous mats. Also, no statistically significant differences in morphological parameters were observed between nanofibers prepared with $10 \mathrm{wt} \%$ gentamicin and without the drug.

\section{Drug release from nanofibrous mats}

Gentamicin release experiments were carried out with multi-layer nanofibrous mats consisting of a crosslinked or noncrosslinked PVA layer of basis weight $0.8 \mathrm{~g} / \mathrm{m}^{2}$ loaded with $10 \mathrm{wt} \%$ gentamicin and outer PUR layers with basis weights of $1.5,4.4$, or $14.7 \mathrm{~g} / \mathrm{m}^{2}$. The concentration of the released gentamicin was assessed using HPLC-MS.

Before starting the gentamicin release experiments, it was crucial to prove the stability of the drug during the incorporation procedure. MS spectra of fresh gentamicin before incorporation were compared with the spectra of gentamicin released from sandwich nanofibers consisting of crosslinked PVA nanofibers and $14.7 \mathrm{~g} / \mathrm{m}^{2}$ PUR covering layers. The mass spectra did not show any differences, suggesting that the structure of gentamicin remained unchanged.

The antimicrobial effectiveness of gentamicin released from crosslinked and noncrosslinked PVA layers as well as from PUR/PVA/PUR sandwiches was evaluated from the inhibition zones of $S$. aureus and $P$. aeruginosa after 1, 2, and 6 days of culture. Clear inhibitory zones were observed with both strains around all of the gentamicin-loaded mats, while no inhibition was found around control nanofibers without the drug. The inhibitory zones on $S$. aureus cultures were comparable with those of gentamicin standard discs that released a similar amount of gentamicin (Figure 2). Smaller inhibitory zones were found on $P$. aeruginosa cultures when compared to $S$. aureus; nevertheless, the inhibitory activity of gentamicin against $P$. aeruginosa was still preserved (Figure 2D).

These tests proved that gentamicin survives "hard" electrospinning conditions, such as exposure to phosphoric acid, a high electrostatic field, and an elevated temperature during PVA crosslinking, in terms of maintaining its structure and antibacterial activity.

The gentamicin release experiments were first carried out by immersing samples of the nanofibrous mats (pieces of ca $0.1 \mathrm{~g})$ in an excess of water $(10 \mathrm{~mL})$. Figure 3 shows 

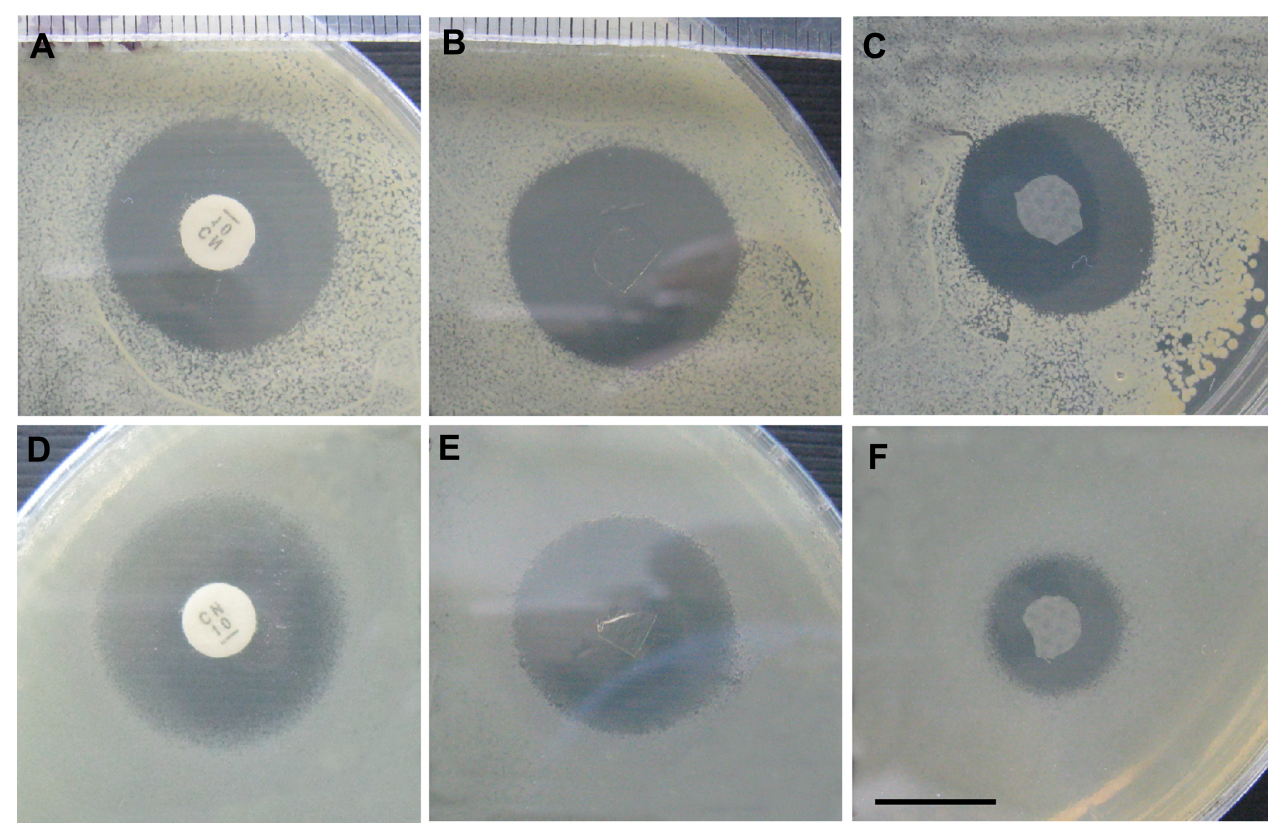

Figure 2 The areas of inhibited growth of Staphylococcus aureus (A-C) and Pseudomonas aeruginosa (D-F) around gentamicin standard discs (A and D), a noncrosslinked PVA layer with 10 wt\% gentamicin (B and E), and a crosslinked PVA layer with 10 wt\% gentamicin (C and F).

Note: Scale: $1 \mathrm{~cm}$.

Abbreviation: PVA, polyvinyl alcohol.

the dependence of the released gentamicin (in percentage) on time. It can be seen that the release of almost $90 \%$ of the total drug amount occurred within 1 hour. An effect of the various thicknesses of the covering PUR layers was observed only for very short exposure times (10 and 30 minutes). For longer exposure times, regardless of the PUR layer thickness, the amount of the released drug was similar $(90 \%-100 \%)$. No significant differences in the release profiles were found by comparing samples with a noncrosslinked or crosslinked PVA layer.

In the case of all tested samples, the fast release can be explained by several factors. During shaking, the flow of a liquid medium is greater and it can easily penetrate through a nanofibrous structure. The middle drug-loaded layer is easily accessible and the release of gentamicin as a watersoluble drug is thus accelerated. Moreover, the excess water and lower gentamicin concentration in solution contribute to a steeper concentration gradient, and, consequently, faster drug diffusion, respectively.

It is necessary to note that this experimental arrangement does not simulate the actual conditions of drug release in potential applications, particularly in wound healing. Therefore, it would be desirable to perform the experiments in such a way that would more closely approximate real drug release into surrounding living tissue. Unfortunately, this aspect has often been neglected by other authors, and most published reports rely on monitoring drug release into a large volume of liquid media. So, in the next step, release experiments were carried out by placing the sample discs on wet agar plates. The antibacterial assays (discussed below) were also done using agar plates. The discs were removed from the agar after various time periods, and the residual gentamicin was washed out into water and quantified by HPLC-MS analysis. The amount of gentamicin released to the agar was calculated from the differences between the initial and the residual amounts.

In the case of samples with gentamicin incorporated into sandwiches with a noncrosslinked PVA layer, the release was generally fast regardless of the thickness of the covering PUR layer. The faster release was influenced by the degradation of the PVA layer and the subsequent easier dissolution of the gentamicin.

Significantly different results were obtained from sandwich samples with a crosslinked PVA layer. The release profiles are depicted in Figure 4. In the case of the thinnest covering PUR nanofibrous layers $\left(1.5 \mathrm{~g} / \mathrm{m}^{2}\right)$, the entire quantity of gentamicin was released within 1 hour of sample application onto the agar plate. There was no apparent difference between the amount of residual gentamicin in nanofibers applied to the agar plate for 1 hour and those applied for any longer time period. A similar gentamicin release profile was observed for crosslinked PVA nanofibers without PUR 

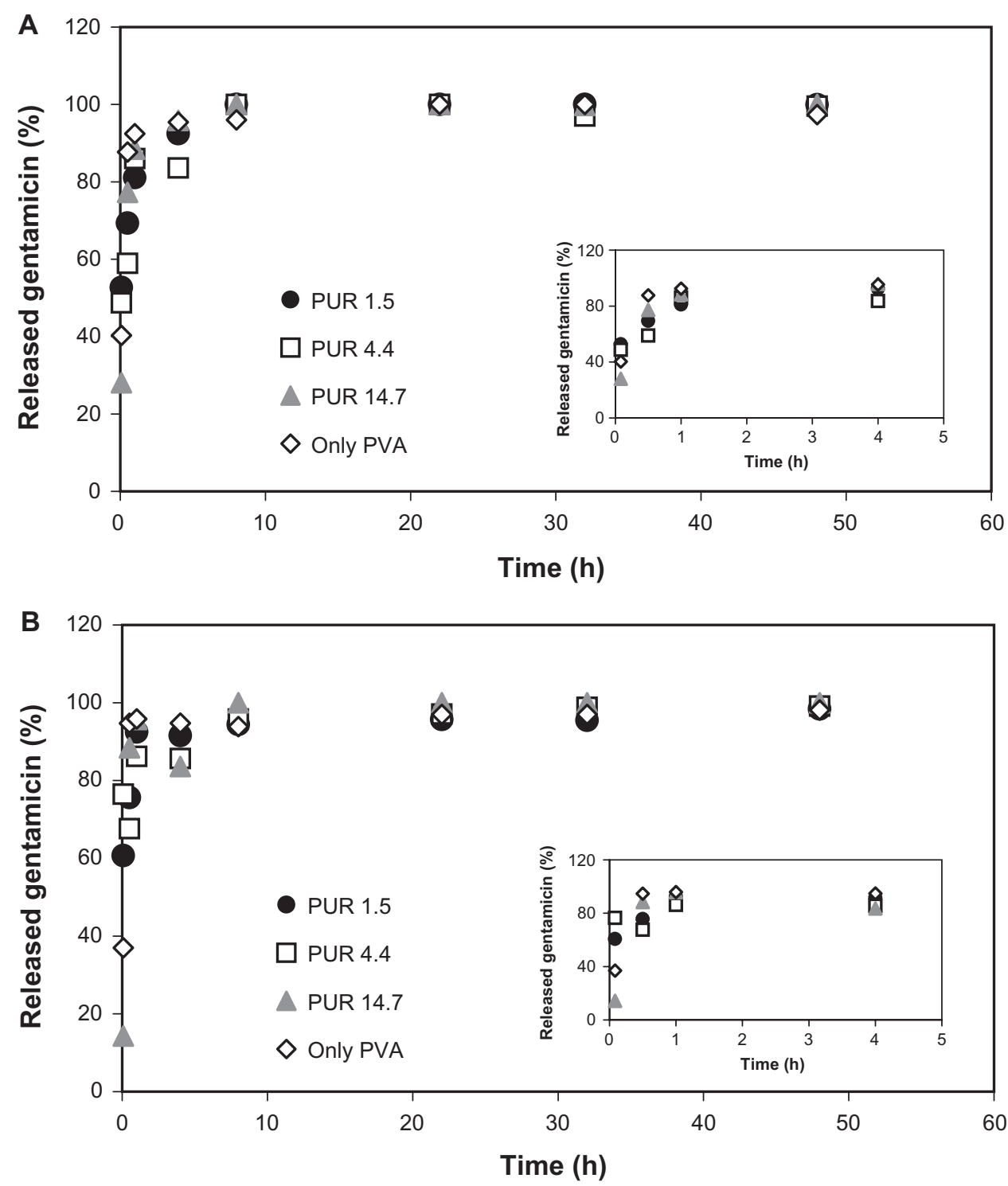

Figure 3 Release profiles of gentamicin into water from PUR/PVA/PUR nanofibrous sandwiches with PUR layers of $1.5,4.4$, or I4.7 g/m² basis weight and a noncrosslinked (A) or crosslinked (B) PVA layer.

Abbreviations: PUR, polyurethane; PVA, polyvinyl alcohol.

layers (not depicted). A significant difference was found in the case of samples covered by $4.4 \mathrm{~g} / \mathrm{m}^{2}$ or $14.7 \mathrm{~g} / \mathrm{m}^{2}$ PUR layers. Sandwiches with $4.4 \mathrm{~g} / \mathrm{m}^{2}$ PUR layers retained about $10 \%$ of the gentamicin after 24 hours of application, while those with $14.7 \mathrm{~g} / \mathrm{m}^{2}$ PUR layers retained $30 \%$ after 24 hours and still contained about $20 \%$ of gentamicin even after 48 hours.

In accordance with the results discussed above, the antibacterial experiments were carried out only with sandwiches based on a crosslinked drug-loaded PVA layer. The gentamicin release profiles were evaluated from the area of inhibition of S. aureus after various time periods (Figure 5). After 1 hour, the largest inhibitory area (85\%) was found for
$1.5 \mathrm{~g} / \mathrm{m}^{2}$ PUR sandwiches, indicating the largest amount of released gentamicin, while the inhibitory zones were smaller around the thicker nanofibrous sandwiches with outer PUR layers of $4.4 \mathrm{~g} / \mathrm{m}^{2}(59 \%)$ or $14.7 \mathrm{~g} / \mathrm{m}^{2}(56 \%)$ (Figure $5 \mathrm{~A}$ ). After 6 hours of treatment, the inhibitory zones achieved their maximal size around all samples and did not increase further with time.

The residual gentamicin in the samples was evaluated by applying the removed discs onto fresh bacterial cultures. No inhibition zones were observed around the $1.5 \mathrm{~g} / \mathrm{m}^{2}$ PUR layered mats, while the inhibition zones confirmed gentamicin retention in the $4.4 \mathrm{~g} / \mathrm{m}^{2}$ and $14.7 \mathrm{~g} / \mathrm{m}^{2}$ PUR layered mats even after 24 hours (Figure 5B). Bacterial inhibition, 


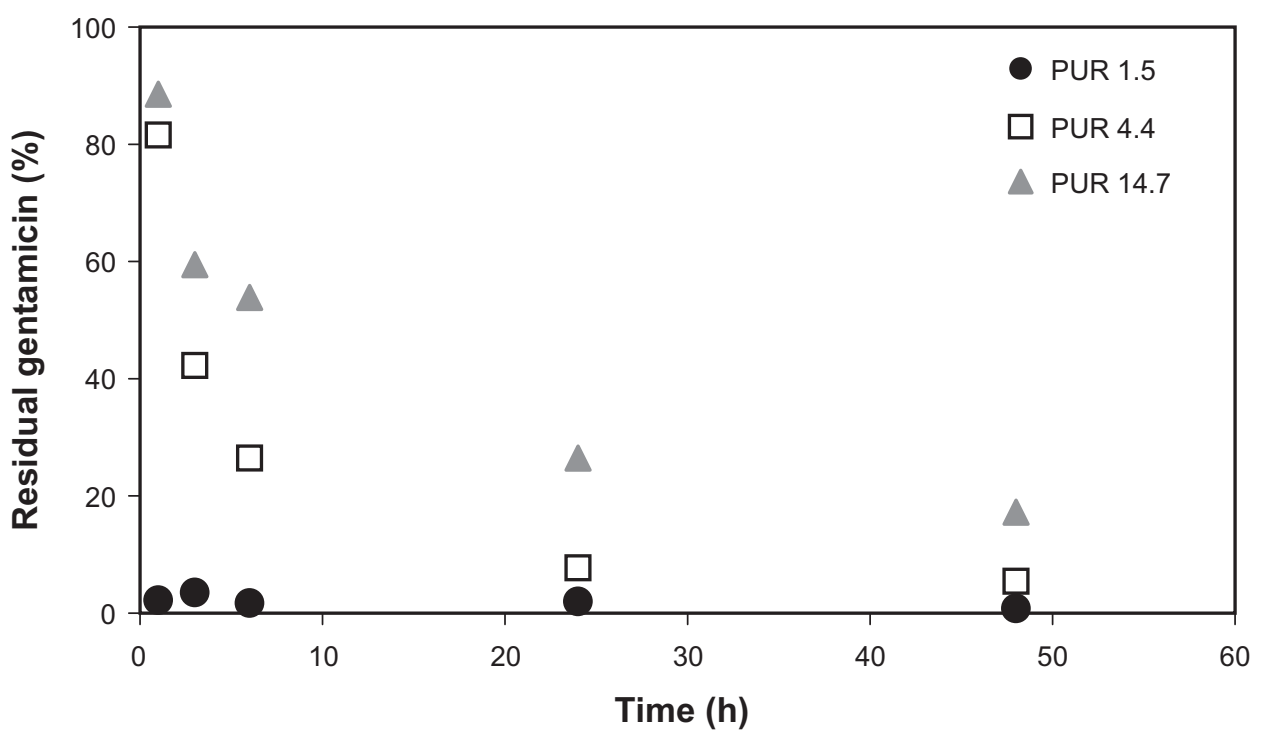

Figure 4 Release profiles of gentamicin to agar from PUR/PVA/PUR nanofibrous sandwiches with crosslinked PVA layers and PUR layers of I.5, 4.4, or I4.7 g/m² basis weights, expressed as the percentage of residual gentamicin remaining in the nanofibrous discs.

Abbreviations: PUR, polyurethane; PVA, polyvinyl alcohol.

however, requires a certain limiting inhibitory concentration of gentamicin, and drug release is therefore not detectable in the more distant areas around the nanofibrous mats where the gentamicin concentration is below the level needed to exert an inhibitory effect.

It can be concluded that the $1.5 \mathrm{~g} / \mathrm{m}^{2}$ PUR layers are too thin to influence the release of gentamicin and to prolong its retention in the nanofibrous mats. Prolonged drug release was found for thicker covering layers of $4.4 \mathrm{~g} / \mathrm{m}^{2}$ or $14.7 \mathrm{~g} / \mathrm{m}^{2}$ thicknesses, but even so, more than $70 \%$ of the gentamicin was released within 24 hours.

The prolongation of the gentamicin release was influenced by two factors: (1) since a covering PUR layer with a higher basis weight is thicker, the gentamicin molecules must travel a longer distance from the surface of the PVA nanofibers to the free volume; and (2) thicker nanofibrous mats are more compact with a lower porosity than thinner ones (see the column "Porosity" in Table 1). Consequently, migrating molecules of the released drug must travel through a nanofibrous layer with smaller pores.

Both these factors are apparently involved in prolonging drug retention. Gibson et $\mathrm{a}^{34}$ studied water vapor diffusion and gas convection through nanofibrous mats and found a linear dependence of flow resistance on the thickness of the nanofibrous mats.

This dependence was also mentioned by Kim et al ${ }^{32}$ in the case of rhodamine and peptide release from multilayered PCL/polyethylene oxide/PCL nanofibers into a liquid environment. Unlike these authors, we have expressed the thickness of the nanofibrous layers in basis weight because, according to our experience, thicknesses expressed in micrometers can vary significantly due to the compression of nanofibrous mats during manipulation or in in vitro/in vivo experiments. Thickness expressed in micrometers is also dependent on the environment - whether the sample is in dry conditions or immersed in water or a saline solution. The values obtained by SEM measurements performed under vacuum/dry conditions may differ considerably from the real thickness of nanofiber mats in water/wet conditions during biological experiments or in medical applications. Obviously, these changes affect the thickness of nanofibers as well as their total porosity and the distribution of the pore sizes, particularly the mean and most frequent pore radius. The release of the incorporated compound is also significantly dependent on the conditions of the experiment - its release into a liquid environment is different when compared to its release into a solid or heterogeneous environment, such as biological tissues in a living organism.

We are aware that a PVA nanofibrous layer with a basis weight of $0.8 \mathrm{~g} / \mathrm{m}^{2}$ and $10 \mathrm{wt} \%$ gentamicin was used to model gentamicin release from sandwiches of various thicknesses, but that this does not correspond to the actual therapeutic dosage of gentamicin commonly used for local application. The required therapeutic dose of gentamicin or other drugs can be easily obtained by adjusting the thickness (basis weight) of the middle drug-loaded layer or by adjusting the drug's concentration in the electrospun mixture. Nevertheless, the thickness of the layers needs to be further optimized to control 

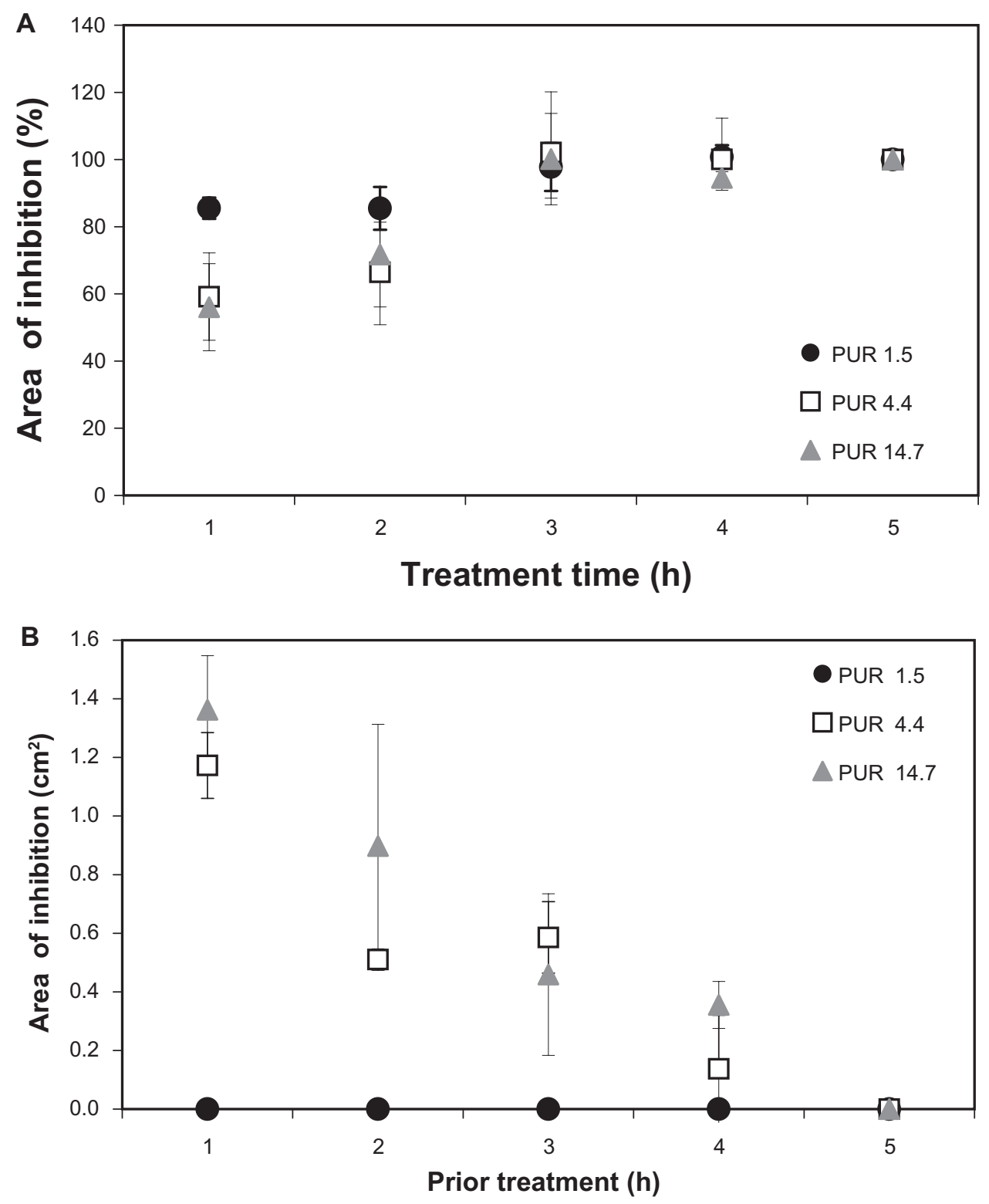

Figure 5 (A) Area of inhibition (\% of the maximal inhibition area after 48 hours) of Staphylococcus aureus agar cultures around nanofibrous samples during gentamicin release. (B) Inhibitory zones from the residual gentamicin in the nanofibrous samples after various intervals of gentamicin release.

the drug release rate and to maintain the mechanical properties or other features of multi-layer nanofibrous mats.

In our previous studies, ${ }^{35,36}$ we demonstrated that nanofibers produced by needleless technology represent a convenient scaffold for cell cultivation and subsequent transfer to damaged tissue. The multilayered nanofibers studied in this work may offer a promising combination of such inherent properties of electrospun nanofibers with controlled drug release for developing suitable scaffolds for wound dressing applications. Moreover, the use of Nanospider ${ }^{\mathrm{TM}}$ technology represents a relatively simple and easily controllable way to fabricate such nanofibrous scaffolds in mass production, which is advantageous when compared to other methods.

\section{Conclusion}

In the present study, needleless electrospinning technology was successfully used for the preparation of PUR/PVA/PUR multi-layer nanofibrous mats with gentamicin incorporated into the middle PVA layer. This method enables effective large-scale production of nanofibrous sandwiches for various medical applications. Morphological characterization of the sandwiches revealed highly homogeneous nanofibrous structures with tight connection between the individual nanofiber layers. HPLC/MS analysis, as well as inhibition of bacterial growth, proved the stability of the gentamicin after its incorporation into the nanofibers during electrospinning. Increasing the thickness of the covering layers prolonged 
gentamicin retention. Nanofibrous multi-layer drug carriers seem to be promising materials for use in various medical applications.

\section{Acknowledgments}

This work was supported by grants: AS CR KAN200520804, SVV 2012265201, and MEYS MSM 0021620857. We thank James Dutt for his critical reading of our manuscript.

\section{Disclosure}

The authors report no conflicts of interest in this work.

\section{References}

1. Vasita R, Katti DS. Nanofibers and their applications in tissue engineering. Int J Nanomedicine. 2006;1(1):15-30.

2. Kubinova S, Sykova E. Nanotechnologies in regenerative medicine. Minim Invasive Ther Allied Technol. 2010;19(3-4):144-156.

3. Khang D, Carpenter J, Chun YW, Pareta R, Webster TJ. Nanotechnology for regenerative medicine. Biomed Microdevices. 2010;12(4): $575-587$.

4. Kumbar SG, Nair LS, Bhattacharyya S, Laurencin CT. Polymeric nanofibers as novel carriers for the delivery of therapeutic molecules. J Nanosci Nanotechnol. 2006;6(9-10):2591-2607.

5. Chandrasekaran AR, Venugopal J, Sundarrajan S, Ramakrishna S. Fabrication of a nanofibrous scaffold with improved bioactivity for culture of human dermal fibroblasts for skin regeneration. Biomed Mater. 2011;6(1):1-10.

6. Noh HK, Lee SW, Kim JM, et al. Electrospinning of chitin nanofibers: Degradation behavior and cellular response to normal human keratinocytes and fibroblasts. Biomaterials. 2006;27(21):3934-3944.

7. Park CJ, Clark SG, Lichtensteiger CA, Jamison RD, Johnson AJW. Accelerated wound closure of pressure ulcers in aged mice by chitosan scaffolds with and without bFGF. Acta Biomater. 2009;5(6): 1926-1936.

8. Rho KS, Jeong L, Lee G, et al. Electrospinning of collagen nanofibers: Effects on the behavior of normal human keratinocytes and early-stage wound healing. Biomaterials. 2006;27(8):1452-1461.

9. Verreck G, Chun I, Rosenblatt J, et al. Incorporation of drugs in an amorphous state into electrospun nanofibers composed of a water-insoluble, nonbiodegradable polymer. J Control Release. 2003;92(3):349-360.

10. Liu X, Lin T, Fang JA, et al. In vivo wound healing and antibacterial performances of electrospun nanofibre membranes. J Biomed Mater Res Part A. 2010;94A(2):499-508.

11. Babaeijandaghi F, Shabani I, Seyedjafari E, et al. Accelerated epidermal regeneration and improved dermal reconstruction achieved by polyethersulfone nanofibers. Tissue Eng Part A. 2010;16(11):3527-3536.

12. Katti DS, Robinson KW, Ko FK, Laurencin CT. Bioresorbable nanofiber-based systems for wound healing and drug delivery: Optimization of fabrication parameters. J Biomed Mater Res Part B. 2004;70B(2):286-296.

13. Thakur RA, Florek CA, Kohn J, Michniak BB. Electrospun nanofibrous polymeric scaffold with targeted drug release profiles for potential application as wound dressing. Int J Phar. 2008;364(1):87-93.

14. Peng HS, Zhou SB, Guo T, et al. In vitro degradation and release profiles for electrospun polymeric fibers containing paracetanol. Colloids Surf B Biointerfaces. 2008;66(2):206-212.

15. Choi JS, Leong KW, Yoo HS. In vivo wound healing of diabetic ulcers using electrospun nanofibers immobilized with human epidermal growth factor (EGF). Biomaterials. 2008;29(5):587-596.
16. Huang Z, Zhang Y, Kotaki M, Ramakrishna S. A review on polymer nanofibers by electrospinning and their applications in nanocomposites. Compos Sci Technol. 2003;63:2223-2253.

17. Martins A, Reis R, Neves N. Electrospinning: processing technique for tissue engineering scaffolding. Int Mater Rev. 2008;53(5):257-274.

18. Bellan L, Craighead H. Applications of controlled electrospinning systems. Polym Adv Technol. 2011;22:304-309.

19. Thompson C, Chase G, Yarin A, Reneker D. Effects of parameters on nanofiber diameter determined from electrospinning model. Polymer. 2007;48(23):6913-6922.

20. Lee GH, Song JC, Yoon KB. Controlled wall thickness and porosity of polymeric hollow nanofibers by coaxial electrospinning. Macromol Res. 2010;18(6):571-576.

21. Wang C, Yan K, Lin Y, Hsieh P. Biodegradable core/shell fibers by coaxial electrospinning: Processing, fiber characterization, and its application in sustained drug release. Macromolecules. 2010;43(15): 6389-6397.

22. Jirsak O, Sanetrnik F, Lukas D, Kotek L, Martinova L, Chaloupek J, inventors; Technical University of Liberec, assignee. Method of nanofibers production from polymer solution using electrostatic spinning and a device for carrying out the method. United States patent US 20060290031. December 28, 2006.

23. Lukas D, Sarkar A, Pokorny P. Self-organization of jets in electrospinning from free liquid surface: A generalized approach. J Appl Phys. 2008;103:No 084309.

24. Barnes CP, Sell SA, Boland ED, Simpson DG, Bowlin GL. Nanofiber technology: Designing the next generation of tissue engineering scaffolds. Adv Drug Deliv Rev. 2007;59(14):1413-1433.

25. Taylor G. Electrically driven jets. Proc R Soc Lond A Math Phys Sci. 1969:453-475.

26. Park JC, inventor; Finetex Ene, Inc, assignee. Electric spinning apparatus for mass-production of nano-fiber. United States patent US 7980838. March 26, 2008.

27. Holan V, Chudickova M, Trosan P, et al. Cyclosporine A-loaded and stem cell-seeded electrospun nanofibers for cell-based therapy and local immunosuppression. J Control Release. 2011;156(3):406-412.

28. Srikar R, Yarin AL, Megaridis CM, Bazilevsky AV, Kelley E. Desorption-limited mechanism of release from polymer nanofibers. Langmuir. 2008;24(3):965-974.

29. Gandhi M, Srikar R, Yarin AL, Megaridis CM, Gemeinhart RA. Mechanistic examination of protein release from polymer nanofibers. Mol Phar. 2009;6(2):641-647.

30. Huang ZM, He CL, Yang AZ, et al. Encapsulating drugs in biodegradable ultrafine fibers through co-axial electrospinning. J Biomed Mater Res Part A. 2006;77A(1):169-179.

31. Chen DWC, Liao JY, Liu SJ, Chan EC. Novel biodegradable sandwichstructured nanofibrous drug-eluting membranes for repair of infected wounds: an in vitro and in vivo study. Int $J$ Nanomedicine. 2012;7: 763-771.

32. Kim G, Yoon H, Park Y. Drug release from various thicknesses of layered mats consisting of electrospun polycaprolactone and polyethylene oxide micro/nanofibers. Appl Phys A Mater Sci Process. 2010;100(4): $1197-1204$.

33. Fathi-Azarbayjani A, Chan SY. Single and multi-layered nanofibers for rapid and controlled drug delivery. Chem Pharm Bull. 2010;58(2): 143-146.

34. Gibson PW, Schreuder-Gibson HL, Rivin D. Electrospun fiber mats: Transport properties. AIChE J. 1999;45(1):190-195.

35. Dubsky M, Kubinova S, Sirc J, et al. Nanofibers prepared by needleless electrospinning technology as scaffolds for wound healing. J Mater Sci Mater Med. 2012;23(4):931-941.

36. Zajicova A, Pokorna K, Lencova A, et al. Treatment of ocular surface injuries by limbal and mesenchymal stem cells growing on nanofiber scaffolds. Cell Transplant. 2010;19(10):1281-1290. 
International Journal of Nanomedicine

Dovepress

\section{Publish your work in this journal}

The International Journal of Nanomedicine is an international, peerreviewed journal focusing on the application of nanotechnology in diagnostics, therapeutics, and drug delivery systems throughou the biomedical field. This journal is indexed on PubMed Central, MedLine, CAS, SciSearch ${ }^{\circledR}$, Current Contents ${ }^{\circledR} /$ Clinical Medicine,
Journal Citation Reports/Science Edition, EMBase, Scopus and the Elsevier Bibliographic databases. The manuscript management system is completely online and includes a very quick and fair peer-review system, which is all easy to use. Visit http://www.dovepress.com/ testimonials.php to read real quotes from published authors.

Submit your manuscript here: http://www.dovepress.com/international-journal-of-nanomedicine-journal 